\title{
Cambio técnico en la industria manufacturera en México, 2003-2008 y 2008-2012
}

Brenda Murtllo-Villanueva

\section{RESUMEN}

Este documento analiza el cambio técnico de los veintiún subsectores de la industria manufacturera en México en 2003-2008 y 2008-2012. El análisis se basa en identificar los tipos de cambio técnico más observados y en ubicar los subsectores más propensos al cambio técnico. El estudio del cambio técnico y su evolución se llevó a cabo a partir de las matrices de insumo-producto de México que permiten observar para cada subsector la evolución de los requerimientos de insumos intermedios y primarios. Los resultados muestran que la mayoría de los subsectores manufactureros han optado en los últimos años por un menor uso del factor trabajo, que se ha acompañado por un uso más intensivo de insumos con mayor contenido tecnológico.

Palabras clave: cambio técnico, insumo-producto, industria manufacturera

Clasificación JEL: O33, C67

Profesora-investigadora de la Facultad de Economía de la Universidad Autónoma del Estado de México, México. Correo electrónico: bmv_17_5@hotmail.com 


\section{ABStract}

Technical Change in the Mexican Manufacturing Industry, 20032008 and 2008-2012

This document analyzes the technical change in the 21 Mexican manufacturing subsectors in 2003-2008 and 2008-2012- The analysis identifies the most observed types of technical change and locates the subsectors prone to technical change. The study of technical change and its evolution was carried out through the Mexican input-output tables since they allow to observe for each subsector the evolution of the intermediate and primary input requirements. The results show that in the last years the majority of manufacturing industries have reduced the use of labor and increased the use of inputs with greater technological content. Key Words: technical change, input-output analysis, manufacturing industry

JEL Classification: O33, C67

\section{INTRODUCCIÓN}

Una de las hipótesis de crecimiento económico más aceptada en los últimos años es la hipótesis del crecimiento económico basado en el progreso técnico. El progreso técnico es la consecuencia del cambio técnico y sucede cuando la productividad de los factores incrementa. Por su parte, el cambio técnico se define como el cambio en la estructura y contenido de la producción, e implica un mejoramiento o un cambio en las dinámicas de producción existentes, en el conjunto de factores que se combinan o en las cantidades de éstos que se utilizan. Varios autores como Schumpeter (1942), Solow (1956), Arrow (1962) y Pasinetti (1983) han argumentado que, a través de la adopción e incorporación de nuevas técnicas y procesos productivos, una economía puede alcanzar niveles superiores de crecimiento e incluso crecimiento sostenido de largo plazo. También se ha encontrado que mediante el progreso técnico los niveles de capital humano y de calificación de la mano de obra son superiores (véase Rosenberg, 2000), lo que evita el atraso de una economía, acelera el ritmo de crecimiento económico y permite un aumento en la productividad del trabajo.

De acuerdo con la teoría clásica, el análisis del cambio técnico es un análisis con perspectiva de largo plazo, principalmente porque en el 
largo plazo todos los factores de producción son variables y porque si se razona en términos de paradigmas económicos (Freeman y Perez, 1988 y Dosi, 1982), es probable que las transformaciones técnicas abarquen periodos de cincuenta años (Kondratieff, 1935). No obstante, este trabajo plantea que las tecnologías de cada paradigma cambian, se combinan y recombinan en lapsos cada vez menores, ocasionando que la estructura productiva de las distintas economías sean más dinámicas.

Por otro lado, diversos autores han destacado la importancia de la dinámica sectorial en la actividad económica; en específico, Kaldor (1966) y Prebisch (1949), entre otros, argumentan que el desempeño del sector manufacturero influye de manera importante en la evolución de la economía nacional; y el análisis de la manufactura de manera desagregada se vuelve relevante.

Lo anterior debido a que el cambio técnico genera diferencias en las tasas de crecimiento de la productividad entre industrias y sectores (véase Lall, 1992, 2004 y Kim, 1997), lo que a su vez trae como consecuencia el rápido crecimiento de industrias que utilizan nuevas técnicas y tecnologías, y a la desaparición de aquellas que utilizan técnicas viejas. Como la aparición de nuevas técnicas no es un proceso suave ni continuo, el cambio técnico puede ser extremadamente desigual a lo largo del tiempo entre industrias y sectores e incluso geográficamente entre países y regiones.

Al respecto, Coombs, Saviotti y Walsh (1987) indican que la persistente dispersión de las tasas de crecimiento de la productividad entre sectores, donde las diferencias entre las tasas de crecimiento son el resultado de diferentes tasas de cambio técnico, está fuertemente relacionada con la capacidad que tienen ciertas industrias de beneficiarse del cambio técnico según sus bases científicas y tecnológicas.

También existe evidencia en el trabajo de De Long y Summers (1991) de que las derramas tecnológicas son mayores en algunos sectores que en otros. Considerando que existen sectores en los que la tasa de crecimiento del progreso técnico es mayor que en otros, Dowrick (1994) señala que un país podría incrementar su tasa de crecimiento conjunta si asigna recursos en los sectores en los que el progreso técnico es más rápido, ya que permiten la continua incorporación de nuevas y mejores técnicas que incrementan la productividad del trabajo y el producto per cápita. El desarrollo y crecimiento de estas industrias clave a su vez facilita el crecimiento del resto del sistema productivo, 
ya que de manera indirecta incrementa los requerimientos de insumos necesarios en su producción.

En ese sentido, este trabajo pretende contribuir en el estudio del cambio técnico a nivel de subsector manufacturero a partir del análisis de la estructura productiva de la manufactura; el objetivo es determinar a través de la medición del cambio en las matrices de coeficientes técnicos de México en dos periodos (2003-2008 y 2008-2012), cuáles fueron los subsectores que mostraron mayores cambios en su estructura productiva. La hipótesis es que en periodos de cinco años, aunque los cambios en la mayoría de los coeficientes técnicos son estables, se logran observar algunos cambios relevantes en el contenido y la estructura productiva de los subsectores manufactureros, principalmente entre insumos primarios e intermedios; la mayoría de los subsectores ha optado por un cambio técnico que reduce el contenido de trabajo.

Para ello, el documento se divide en 3 apartados además de la introducción y las conclusiones. En el primero se presenta la metodología de insumo-producto, que nos permitirá observar el cambio técnico de los 21 subsectores de la manufactura. El segundo apartado analiza el cambio técnico en dos etapas; en la primera se compara la intensidad de uso de los insumos intermedios y primarios, y en la segunda se analizan a detalle la evolución en el uso de los insumos intermedios. En el tercer apartado se analizan los efectos fabricación y sustitución que explican el cambio en la estructura productiva.

\section{Metodología}

Las matrices de insumo-producto son tablas de doble entrada que registran las relaciones entre los distintos sectores de una economía necesarias para la producción de bienes y servicios. Dichas relaciones obedecen a las fuerzas de oferta y demanda, y permiten observar la estructura productiva de una economía y la técnica de producción de las distintas industrias.

Desde la perspectiva de la oferta global(OG), las matrices de insumoproducto dan cuenta de la adquisición de insumos para la producción, los cuales pueden ser intermedios de origen interno $\left(\mathrm{X}_{\mathrm{nj}}\right)$ o importado $\left(\mathrm{M}_{\mathrm{nj}}\right)$, primarios como el trabajo $\left(\mathrm{W}_{\mathrm{j}}\right)$ y el capital $\left(\mathrm{P}_{\mathrm{j}}\right)$, y el pago de impuestos netos a la producción $\left(\mathrm{NT}_{\mathrm{j}}\right)$ (ecuación 1$)$. Los insumos intermedios representan todos los insumos provenientes de otros sectores 
que son requeridos e incorporados en el bien producido por el sector en cuestión y los cuáles desaparecen al terminar el proceso productivo (capital circulante), mientras que los insumos primarios representan a los factores productivos trabajo y capital, los cuáles perduran, aunque probablemente no en su totalidad, después del proceso productivo.

Esta relación de interdependencia de la oferta global (OG) también puede expresarse como la suma de la compra de insumos intermedios, llámese consumo intermedio interno e importado $\left(\sum_{\mathrm{i}=1}^{\mathrm{n}} \mathrm{X}_{\mathrm{ij}}+\sum_{\mathrm{i}=1}^{\mathrm{n}} \mathrm{M}_{\mathrm{ij}}\right), \mathrm{y}$ de la suma de la compra de insumos primarios, llámese valor agregado $\left(V A_{j}\right)$ (ver ecuación 2).

$$
\begin{aligned}
& O G=V B P_{j}=X_{j}=X_{1 j}+X_{2 j}+\cdots+X_{n j}+M_{1 j}+M_{2 j}+\cdots+M_{n j}+W_{j}+P_{j}+N T_{j} \\
& X_{j}=\sum_{i=1}^{n} X_{i j}+\sum_{i=1}^{n} M_{i j}+V A_{j}, \text { donde } V A_{j}=W_{j}+P_{j}+N T_{j}
\end{aligned}
$$

La información sobre las relaciones de interdependencia se representa de dos formas, una es a través de la matriz interna de insumo-producto y la otra, a través de la matriz total ${ }^{2}$. Considerando que el núcleo de esta investigación es conocer el cambio en las técnicas de producción a nivel sectorial, se utilizan las matrices totales de insumo-producto ya que consideran que los insumos para la producción pueden ser nacionales o importados.

La técnica con la que producen las industrias se conoce a partir del análisis de los coeficientes directos de insumos intermedios (también llamados coeficientes técnicos) y de los coeficientes de insumos primarios, que se obtienen al dividir las celdas de cada columna por el valor total de la misma (ver cuadro 1 ).

2 La diferencia entre ellas reside en el tratamiento que se da a las importaciones; la matriz interna registra de manera agregada en un vector fila la adquisición de insumos intermedios importados, mientras que la matriz total incorpora de manera desagregada y por industria las importaciones de insumos intermedios; la matriz total resulta de la suma de la matriz interna y la matriz de importaciones. La decisión de utilizar la matriz interna o la matriz total dependerá de los objetivos del investigador. 
Cuadro 1

TÉCNICA DE PRODUCCIÓN

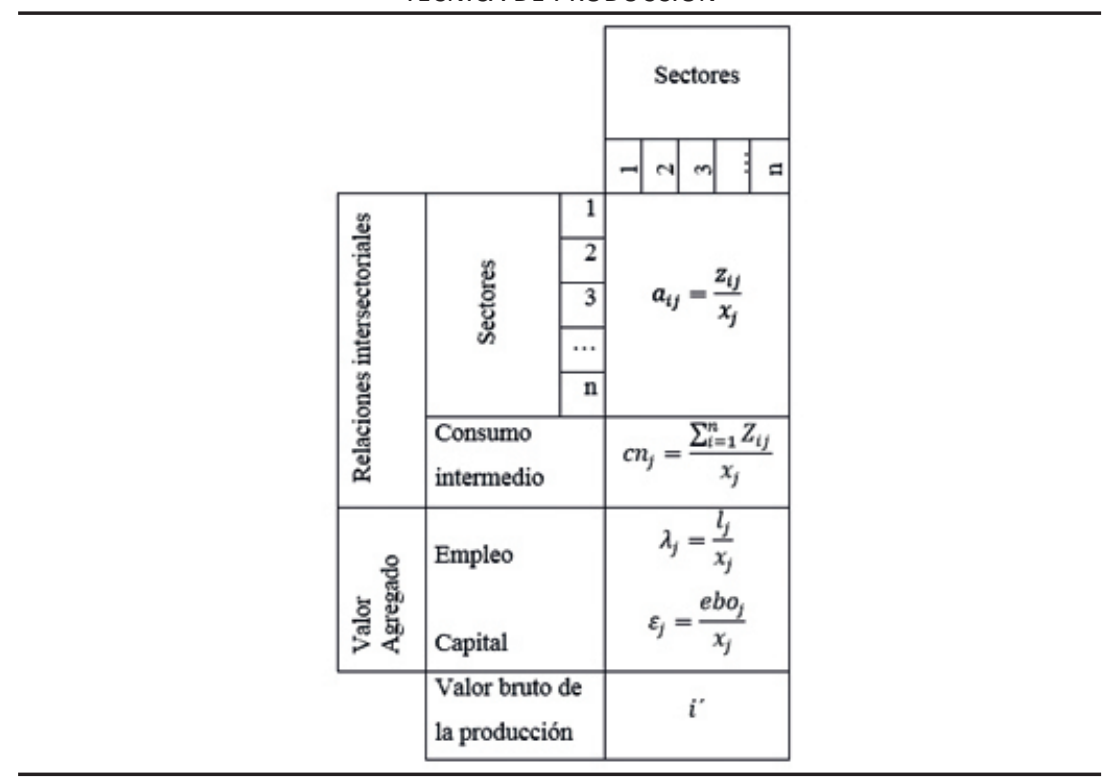

Fuente: elaboración del autor.

Los coeficientes de los insumos primarios (componentes del valor agregado $V A$ ) muestran la intensidad con la que se utiliza el empleo o el capital; el coeficiente de empleo $\left(\lambda_{\mathrm{j}}\right)$ muestra el número de trabajadores utilizados por unidad de producto y el coeficiente ( $(\varepsilon j)$ muestra el excedente bruto de operación por unidad de producto.

Si se cuenta con más de una matriz de insumo-producto es posible medir el cambio en la técnica de producción. Para ello se compara la técnica de producción de cada subsector en el tiempo, y se requiere: a) que los flujos que registran los intercambios entre unidades económicas se descompongan en la cantidad intercambiada y su precio, o $b$ ) que los valores de los intercambios estén a los mismos precios para cada año analizado. Esto permitirá aislar los cambios en los precios e interpretar el cambio en los coeficientes técnicos como el cambio en la técnica de producción (ver Carter, 1970 y Vaccara, 1970). Para este trabajo, se utilizan tres matrices de insumo-producto ${ }^{3}$ correspondientes a los

3 Considerando el gran tamaño de las matrices, estas se encuentran disponibles bajo solicitud directa al autor. 
años 2003, 2008 y 2012 elaboradas por el INEGI (2018); las tres están valuadas a precios básicos del $2008^{4}$ y consideran el mismo sistema de clasificación industrial (SCIAN, 2007) (ver anexo 1).

El cambio en los coeficientes técnicos se debe a diversas razones: cambios tecnológicos, cambios en la calidad de los insumos, en el precio relativo de los insumos intermedios y primarios, economías de escala, entre otros (Vaccara, 1970). Sin embargo, Carter (1970) argumenta que una porción importante del cambio en la estructura se debe a la asimilación de nuevas técnicas. El cambio técnico se observa cuando se experimentan cambios en la manera en que el conjunto de los factores de producción son combinados, cuando la proporción en la que participan los componentes de la técnica de producción se ve alterada o cuando la intensidad con la que se usan los insumos cambia. Este efecto también es resultado del aumento en la gama de materiales y procesos productivos que tiene como consecuencia la sustitución de viejos insumos por nuevos de mejor calidad o cualidades.

\section{EL CAMBIO TÉCNICO EN LA INDUSTRIA MANUFACTURERA EN MÉXICO}

En este trabajo se considera que el cambio técnico es observable en dos etapas; en la primera se identifica la intensidad con que se usan los insumos intermedios en comparación con los insumos primarios (específicamente el empleo), y en la segunda, se analizan los cambios en el consumo de los distintos insumos intermedios. La primera se analiza en el apartado 2.1 y la segunda en el 2.2.

\subsection{Tipo de cambio técnico}

Para la primera etapa se obtienen las diferencias entre periodos de los coeficientes de consumo intermedio y de empleo como se indica en las ecuaciones 3 y 4 . Donde: $\mathrm{d}^{\mathrm{cn}}$ denota la diferencia en el tiempo del coeficiente de consumo intermedio y $c n_{j}$ el coeficiente de consumo intermedio, que es igual a $\sum_{\mathrm{i}=1}^{\mathrm{n}} \mathrm{a}_{\mathrm{ij}}$.

4 Las matrices de 2008 y 2012 fueron construidas por el INEGI a precios básicos de 2008. La matriz de 2003 está valuada a precios de 2003 por lo que fue necesaria su actualización a través del método RAS (ver Murillo-Villanueva, Puchet Anyul y Fujii-Gambero, 2019) 


$$
d^{c n}=c n_{j, t}-c n_{j, t-1}=\sum_{i=1}^{n} a_{i j, t}-\sum_{i=1}^{n} a_{i j, t-1}
$$

Un valor positivo en $\mathrm{d}^{\mathrm{cn}}$ indica que el uso de insumos intermedios ha incrementado en el periodo analizado, es decir, que el sector $\mathrm{j}$ ha optado por aumentar la intensidad con la que se utilizan los insumos provenientes de otras industrias. Este cambio puede asociarse con una reducción en el uso de insumos primarios, o dicho de otra forma, con la sustitución de insumos primarios por insumos intermedios. El cambio en el coeficiente de uso de empleo se conoce a partir de la diferencia en su valor entre periodos (ecuación 4).

$$
d^{\lambda}=\lambda_{t}-\lambda_{t-1}=\frac{l_{j, t}}{x_{j, t}}-\frac{l_{j, t-1}}{x_{j, t-1}}
$$

De los cambios observados en los 21 subsectores manufactureros se determinará que si el cambio en $c n_{\mathrm{i}}$ entre $t$ y $t-1$ es positivo, entonces el uso de los insumos primarios mostró una reducción relativa, ocasionando mayor especialización, ya que se sugiere un uso más eficiente de los insumos primarios. Por el contrario, si el cambio en $c n_{\mathrm{i}}$ entre $t$ y $t-1$ es negativo, el uso de los insumos primarios aumentó en comparación con el uso de insumos intermedios. Las gráficas 1 y 2 muestran para los 21 subsectores manufactureros el crecimiento del coeficiente de los insumos intermedios (eje $\mathrm{x}$ ) y del coeficiente de empleo (eje y), donde cada punto representa un subsector ${ }^{5}$. Como se observa, los puntos se encuentran dispersos y el rango de crecimiento del coeficiente de uso de insumos intermedios es de menor magnitud que el rango de crecimiento del empleo. El cambio técnico experimentado por los subsectores manufactureros lo podemos clasificar en 4 tipos según el crecimiento en el coeficiente de empleo y en el coeficiente de uso de insumos intermedios, los cuales se representan mediante los 4 cuadrantes de las gráficas 1 y 2.

- Tipo 1 (subsectores del cuadrante 1): cambio técnico en el que tanto el uso del empleo como de insumos intermedios se incrementaron, esta situación debe verse compensada con una reducción en el coeficiente de uso de capital fijo.

\footnotetext{
${ }^{5}$ La clasificación SCIAN se muestra de forma detallada en el anexo 1.
} 
- Tipo 2 (subsectores del cuadrante 2): cambio técnico en el que la reducción del coeficiente de uso del empleo se compensó, en todo o en parte, con un aumento en el uso de los insumos intermedios, es un cambio técnico ahorrador de empleo pero intensivo en insumos intermedios.

- Tipo 3 (subsectores del cuadrante 3): cambio técnico en el sentido de que una reducción en el coeficiente de uso del empleo estuvo acompañada de una reducción en el uso de insumos intermedios, lo cual deberá verse compensado con un aumento en el coeficiente de uso de capital fijo.

- Tipo 4 (subsectores del cuadrante 4): cambio técnico en el que se hace mayor uso del factor trabajo y que se compensa, en todo o en parte, con una reducción en el uso de insumos intermedios, este cambio técnico es intensivo en empleo y ahorrador de insumos intermedios.

La gráfica 1 muestra que entre 2003 y 2008 la mayoría de los subsectores mostraron cambio técnico tipo 2, esto quiere decir que la mayoría de industrias optó por una reducción en el uso de empleo que se compensó con un aumento en el coeficiente de insumos intermedios. De manera específica, estos subsectores fueron ahorradores de empleo a través del uso más intensivo de insumos intermedios; podemos observar que hay subsectores como el de fabricación de productos a base de minerales no metálicos (327) con la mayor reducción en el coeficiente de empleo (-13.3\%) y subsectores como el de fabricación de equipo de computación, comunicación, etc. (334) con el mayor incremento en el coeficiente de usos intermedios (1.75\%).

El segundo tipo de cambio técnico más observado es el 3, que se caracteriza por mostrar una reducción tanto en el uso del factor trabajo como en el de insumos intermedios, y que probablemente se compensa con un aumento en el uso del capital; en esta situación destacan la fabricación de prendas de vestir (315) por mostrar una reducción en el uso del empleo en poco más del $-10 \%$ y de los insumos intermedios en casi $-2 \%$, y la fabricación de productos textiles (314) por reducir sus requerimientos de empleo en más del $-12 \%$. 
Gráfica 1

TASAS DE CRECIMIENTO PROMEDIO ANUAL DEL COEFICIENTE DE USO DE EMPLEO Y DEL USO DE INSUMOS INTERMEDIOS, 2003-2008

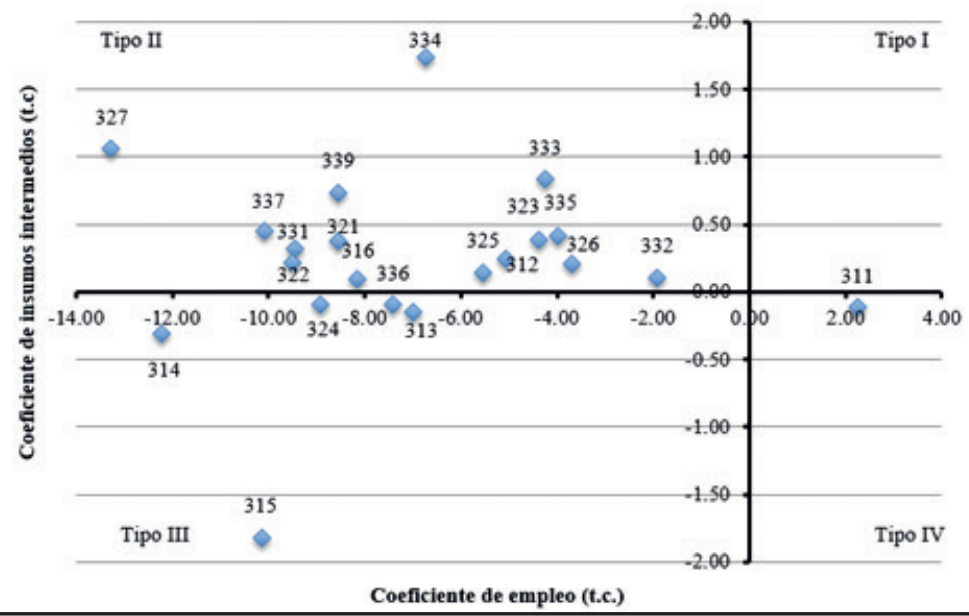

Fuente: elaboración del autor con datos de las MIP de 2003 y 2008 (INEGI, 2018)

Lo anterior indica que entre 2003 y 2008, 20 de los 21 subsectores manufactureros optaron por un cambio técnico ahorrador de empleo. En algunos casos, se acompañó con una reducción en el uso de insumos intermedios, pero en otros se compensó con el mayor uso de estos. Para el primer periodo también se encuentra que no hubo ningún subsector que experimentara cambio técnico del tipo 1 y que de los 21 subsectores únicamente la industria alimentaria (311) aplicó cambio técnico tipo 4 en donde el aumento en el uso de empleo se compensó con una reducción del consumo de insumos intermedios.

Para el periodo 2008-2012 la mayoría de los subsectores mostraron cambio técnico tipo 3 (ver gráfica 2), el segundo cambio técnico más observado fue el tipo $2 \mathrm{y}$, a diferencia del primer periodo, se observa un subsector con cambio técnico tipo 1 y otro con cambio técnico tipo 4. Esto indica que para el segundo periodo, 19 de los 21 subsectores optaron por un cambio técnico ahorrador de empleo, de los cuales 7 compensaron la reducción en el uso del empleo con el aumento en el uso de insumos intermedios y 12 lo acompañaron con un menor uso de éstos.

Dentro de los subsectores que destacan se encuentran: con cambio técnico tipo 2, fabricación de maquinaria y equipo (333) al mostrar un 
incremento en el uso de insumos intermedios en casi 5\%, con cambio técnico tipo 3 fabricación de equipo de computación, comunicación, etc. (334) que para este periodo fue el subsector con la mayor reducción en el uso de insumos intermedios $(-0.75 \%)$, y con cambio técnico tipo 4 la industria química (325) con el mayor aumento en el coeficiente de empleo $(5.1 \%)$ y con la mayor reducción en el uso de insumos interme$\operatorname{dios}(-2.5 \%)$.

\section{Gráfica 2}

TASAS DE CRECIMIENTO PROMEDIO ANUAL DEL COEFICIENTE DE USO DE EMPLEO Y DEL USO DE INSUMOS INTERMEDIOS, 2008-2012

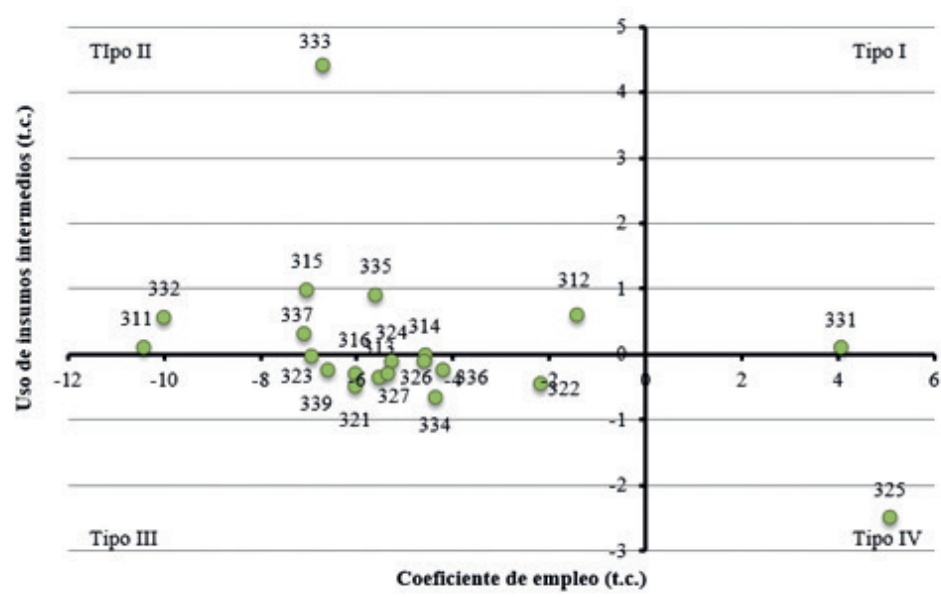

Fuente: elaboración del autor con datos de las MIP de 2008 y 2012 (INEGI, 2018).

En cuanto a los dos periodos analizados, se observan algunos subsectores con características muy definidas. Por ejemplo, los subsectores (313), (314), (324) y (336) mostraron cambio técnico tipo 3 en ambos periodos, y los subsectores (333), (335) y (337) cambio técnico tipo 2. Los primeros muestran un cambio técnico que sustituye el uso de empleo e insumos intermedios por el del capital. Los segundos también han reducido en los 9 años de estudio el uso del trabajo pero lo han compensado con un mayor uso de insumos intermedios. Ambos casos implican un cambio técnico en el que se reduce el contenido de empleo, lo cuál puede relacionarse con un incremento en la productividad laboral. 
Por último, el hecho de que en ambos periodos más del $90 \%$ de los subsectores haya optado por reducir el uso del empleo (cambio técnico tipo 2 y 3) confirma los hallazgos de diversos autores (Dussel-Peters, 2003, Sánchez, 2012, Dussel-Peters y Ortíz, 2013, entre otros) sobre el hecho de que la industria manufacturera ha perdido poder para generar empleo, este trabajo sugiere que ello es así por cuestiones técnicas ya que los resultados muestran que la gran mayoría de subsectores han buscado la forma de reducir el uso de empleo por unidad de producto.

Aunado a esto, considerando que la mayoría de esos subsectores tienen la característica de ser exportadores pero que son los que menos empleo generan por unidad de producto, debido principalmente al bajo coeficiente de empleo que han alcanzado en los últimos años (ver Murillo-Villanueva, Puchet-Anyul y Fujii-Gambero, 2018), y que la mayor parte de los insumos requeridos para su producción es importada (Fujii-Gambero y Cervantes, 2013, Ruíz-Nápoles, 2004), es evidente que la generación de empleo depende de la capacidad para exportar, producir y del fortalecimiento de los vínculos y encadenamientos productivos internos.

\subsection{El cambio en los requerimientos de insumos intermedios}

En la segunda etapa del cambio técnico se profundiza en el análisis de los cambios en el coeficiente de consumo intermedio de cada subsector y se identifica de manera clara los principales cambios en las cantidades de insumos intermedios que utiliza cada subsector y que son proveídos por los distintos subsectores de la economía. La evolución de los coeficientes de uso se observa mediante la diferencia en la magnitud de cada uno de los coeficientes técnicos entre pares de matrices. En este ejercicio, se obtienen dos matrices de diferencias que surgen de las diferencias entre los coeficientes técnicos de la matriz en $t$ y $t-1$ como en las ecuaciones 5 y 6 .

$$
\begin{aligned}
& D_{1}=A_{2008}-A_{2003} \\
& D_{2}=A_{2012}-A_{2008}
\end{aligned}
$$

Si el cambio observado en los coeficientes $\mathrm{a}_{\mathrm{ij}}$ de las matrices $A$ es positivo, entonces, la intensidad con la que se usa el insumo en cuestión 
aumentó, esto puede ser resultado de la sustitución entre insumos, ya que el aumento en un coeficiente inevitablemente se refleja en la disminución de otro (Carter, 1970). El cambio en el valor de los coeficientes técnicos también sugiere cambios técnicos, los cuales se observan cada vez que hay un cambio (adición o eliminación) en los componentes que participan en la técnica de producción. En ese sentido, las entradas de las matrices de diferencias se clasifican según la magnitud de su variación en 7 categorías: no cambio, pequeño, medio y amplio cambio, positivo o negativo. La primer categoría representa una diferencia en cada coeficiente igual a cero y se representa con un número 0 . Las segundas, los cambios que oscilan entre 0 y 0.01 , y entre 0 y -0.01 se señalan con un 1 y un -1 respectivamente. Las terceras, los cambios que oscilan entre 0.011 y 0.1 , y entre -0.011 y -0.1 se indican con un 2 y -2 respectivamente. Y las últimas correspondientes a aquellos cambios mayores a 0.1 y menores a -0.1 se evidencian con un 3 y un -3 respectivamente.

La reducción en los coeficientes técnicos implica ya sea un ahorro en la cantidad de insumos intermedios que se utilizan en comparación con los insumos primarios o la sustitución de ciertos insumos intermedios por otros. La consecuencia de los cambios en la estructura de producción suele ocasionar que la cantidad de producto que antes se obtenía con unas disponibilidades determinadas de factores, resulte aumentada (véase Molins Codina, 1973).

Además, al conocer el sentido de la variación promedio conoceremos el sentido del cambio técnico que ocurre en cada subsector. Aquellos subsectores que en promedio hayan mostrado una disminución en el valor de sus coeficientes técnicos, reflejarán aumentos en la productividad, ya que ahora se consigue el mismo volumen de producción haciendo menor uso del insumo en cuestión, también puede reflejar la sustitución entre insumos intermedios y primarios. Por ejemplo, la reducción en el coeficiente de insumos intermedios refleja ya sea una tendencia generalizada a la baja en los coeficientes técnicos del subsector o una reducción importante en uno o algunos de los coeficientes.

En ese sentido, en este apartado se ofrece una explicación a los más altos cambios en los requerimientos de insumos intermedios, se observa cuáles han sido los insumos que se han dejado de consumir o los que se han consumido con mayor intensidad según sea el caso. 
De manera agregada, los resultados muestran que entre 2003 y 2008 y entre 2008 y 2012 los coeficientes técnicos de los 21 subsectores manufactureros permaneció mayormente estable (ver cuadros 2 y 3 ), lo que sugiere que para la industria manufacturera los grandes cambios en los coeficientes técnicos suelen presentarse en periodos de largo plazo.

Sin embargo, también es importante hacer notar que para casi todos los subsectores manufactureros, los cambios de magnitud media y alta que se captan en periodos de entre 4 y 5 años, se presentan principalmente en las relaciones de cada subsector con el resto de los subsectores manufactureros. Esto indica que las relaciones intersectoriales son más dinámicas en la industria manufacturera y que el cambio técnico se origina mayormente entre ellas. Por otro lado, los resultados de los cuadros 2 y 3 también indican que el cambio técnico fue mucho más dinámico en el primer periodo que en el segundo.

\section{Cuadro 2}

COEFICIENTES TÉCNICOS: MAGNITUD DE VARIACIÓN, 2003-2008*

\begin{tabular}{|c|c|c|c|c|c|}
\hline SCIAN & $\begin{array}{c}\text { Sin cambio } \\
(0)\end{array}$ & 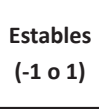 & $\begin{array}{l}\text { Cambio } \\
\text { Mediano } \\
\left(\begin{array}{lll}-2 & 0 & 2\end{array}\right)\end{array}$ & $\begin{array}{c}\text { Alto cambio } \\
(-3 \circ 3)\end{array}$ & $\begin{array}{l}\text { Porcentaje de los cambios de } \\
\text { magnitud media y alta que se } \\
\text { originan en la manufactura }\end{array}$ \\
\hline 311 & 4 & 72 & 2 & 0 & $50 \%$ \\
\hline 312 & 4 & 68 & 6 & 0 & $50 \%$ \\
\hline 313 & 3 & 65 & 10 & 0 & $33 \%$ \\
\hline 314 & 4 & 68 & 6 & 0 & $83 \%$ \\
\hline 315 & 4 & 70 & 4 & 0 & $100 \%$ \\
\hline 316 & 4 & 68 & 6 & 0 & $50 \%$ \\
\hline 321 & 4 & 72 & 2 & 0 & $50 \%$ \\
\hline 322 & 4 & 70 & 4 & 0 & $50 \%$ \\
\hline 323 & 5 & 67 & 6 & 0 & $50 \%$ \\
\hline 324 & 5 & 67 & 5 & 1 & $50 \%$ \\
\hline 325 & 3 & 72 & 3 & 0 & $67 \%$ \\
\hline 326 & 4 & 67 & 7 & 0 & $57 \%$ \\
\hline 327 & 4 & 70 & 4 & 0 & $75 \%$ \\
\hline 331 & 5 & 67 & 6 & 0 & $67 \%$ \\
\hline 332 & 4 & 72 & 2 & 0 & $100 \%$ \\
\hline 333 & 4 & 69 & 5 & 0 & $80 \%$ \\
\hline 334 & 5 & 64 & 8 & 1 & $78 \%$ \\
\hline 335 & 4 & 72 & 2 & 0 & $100 \%$ \\
\hline 336 & 3 & 70 & 5 & 0 & $80 \%$ \\
\hline 337 & 4 & 71 & 3 & 0 & $67 \%$ \\
\hline 339 & 4 & 65 & 9 & 0 & $78 \%$ \\
\hline
\end{tabular}

*Nota: los números representan los coeficientes técnicos de un total 78 para cada subsector. Fuente: elaboración del autor con datos de las MIP de 2008 y 2012 (INEGI, 2018). 
De manera específica, encontramos que para el periodo 2003-2008 (cuadro 2) los subsectores fabricación de equipo de computación, comunicación, etc. (334) y otras industrias manufactureras (339) mostraron el mayor número de coeficientes técnicos con variaciones de magnitud media y alta, y que a su vez, casi el $80 \%$ de estos cambios se observaron en las relaciones con los subsectores manufactureros. También se observa que el subsector fabricación de prendas de vestir (315) es el subsector con el mayor número de coeficientes de magnitud media y alta que se explican en su totalidad por la industria manufacturera. Entre 2008 y 2012, es evidente que los coeficientes técnicos con variación de magnitud media y alta es considerablemente menor, lo que indica que la técnica de producción de los subsectores manufactureros fue aún más estable en el segundo periodo (ver cuadro 3). Sobresalen los subsectores fabricación de productos textiles (314), industrias metálicas básicas (331) y fabricación de maquinaria y equipo (333) por registrar los mayores coeficientes con cambios de magnitud media y alta.

\section{Cuadro 3}

COEFICIENTES TÉCNICOS: MAGNITUd DE VARIACIÓN, 2008-2012*

\begin{tabular}{|c|c|c|c|c|c|}
\hline SCIAN & $\begin{array}{c}\text { Sin cambio } \\
(0)\end{array}$ & $\begin{array}{c}\text { Estables } \\
\left(\begin{array}{l}-1 \circ 1)\end{array}\right.\end{array}$ & $\begin{array}{l}\text { Cambio } \\
\text { Mediano } \\
\left(\begin{array}{lll}-2 & \circ & 2\end{array}\right)\end{array}$ & $\begin{array}{c}\text { Alto cambio } \\
(-3 \circ 3)\end{array}$ & $\begin{array}{l}\text { Porcentaje de los cambios de } \\
\text { magnitud media y alta que se } \\
\text { originan en la manufactura }\end{array}$ \\
\hline 311 & 3 & 75 & 0 & 0 & - \\
\hline 312 & 7 & 70 & 1 & 0 & 100 \\
\hline 313 & 5 & 70 & 3 & 0 & 33 \\
\hline 314 & 5 & 67 & 6 & 0 & 83 \\
\hline 315 & 5 & 71 & 2 & 0 & 100 \\
\hline 316 & 5 & 71 & 2 & 0 & 50 \\
\hline 321 & 4 & 70 & 4 & 0 & 25 \\
\hline 322 & 4 & 73 & 1 & 0 & 100 \\
\hline 323 & 5 & 71 & 2 & 0 & 100 \\
\hline 324 & 6 & 69 & 3 & 0 & 33 \\
\hline 325 & 3 & 71 & 4 & 0 & 25 \\
\hline 326 & 4 & 72 & 2 & 0 & 50 \\
\hline 327 & 4 & 72 & 2 & 0 & 50 \\
\hline 331 & 6 & 68 & 4 & 0 & 25 \\
\hline 332 & 5 & 73 & 0 & 0 & - \\
\hline 333 & 4 & 68 & 6 & 0 & 83 \\
\hline 334 & 5 & 71 & 2 & 0 & 100 \\
\hline 335 & 5 & 69 & 4 & 0 & 75 \\
\hline 336 & 5 & 69 & 4 & 0 & 100 \\
\hline 337 & 6 & 72 & 0 & 0 & - \\
\hline 339 & 3 & 69 & 6 & 0 & 83 \\
\hline
\end{tabular}

*Nota: los números representan los coeficientes técnicos de un total 78 para cada subsector.

Fuente: elaboración del autor con datos de las MIP de 2008 y 2012 (INEGI, 2018). 
Los cuadros 4 y 5 muestran respectivamente para los periodos 20032008 y 2008-2012, las diferencias entre las matrices de coeficientes técnicos de la parte de los vectores columna que corresponde a los subsectores manufactureros, es decir, de los 21 subsectores de la manufactura que ocupan las posiciones del 14 al 34 y del 311 al 339 en la clasificación SCIAN. Con un recuadro se representan las diferencias cuya magnitud se encuentra en los rangos señalados por el 2 y el -2 , y en un recuadro sombreado en gris las diferencias de mayor magnitud señalados por el 3 y el -3. En los anexos 2 y 3 se presenta la información completa para los subsectores manufactureros con el mismo formato.

Las últimas filas de los cuadros 4 y 5 muestran el número de coeficientes técnicos con variaciones de alta magnitud (3 y -3) y magnitud media ( 2 y -2) que se originaron en las relaciones entre los subsectores manufactureros; en estas filas, los subsectores sombreados con color blanco y con un asterisco corresponden a aquellos con cambio técnico tipo 1 , en blanco los de cambio técnico tipo 2, en gris los de cambio técnico tipo 3 y en negro los de cambio técnico tipo 4. Asimismo, las celdas que en estas filas contienen un rombo destacan el sentido y la magnitud de los subsectores con los cambios más relevantes.

En el análisis de la sección anterior, se identificó que para el periodo 2003-2008 los subsectores con los mayores cambios en los requerimientos de insumos intermedios fueron: fabricación de equipo de computación, comunicación, etc. (334) y otras industrias manufactureras (339) con el mayor incremento y fabricación de prendas de vestir (315) con la mayor reducción.

Del cuadro 4 se puede notar que el incremento en el uso de insumos intermedios del subsector fabricación de equipo de computación, comunicación, etc. (334) se explica mayormente por un uso más intensivo de componentes proveídos por el mismo subsector; también se explica, aunque en menor magnitud, por los insumos provenientes del subsector de fabricación de maquinaria y equipo (333), de otras manufacturas (339) y de servicios profesionales, cientificos y técnicos (541). Esto sugiere que el subsector (334) aplicó cambio técnico intensivo en bienes tecnológicos y físicos, lo cual justifica la fuerte caída en el uso del factor trabajo mostrado en la gráfica 1 y el posible incremento en la productividad laboral. Es decir, este sector está encaminado a la tecnificación y a la mejora de los procesos productivos y a la sustitución de empleo por equipo de computación, comunicación, maquinaria y equipo. 


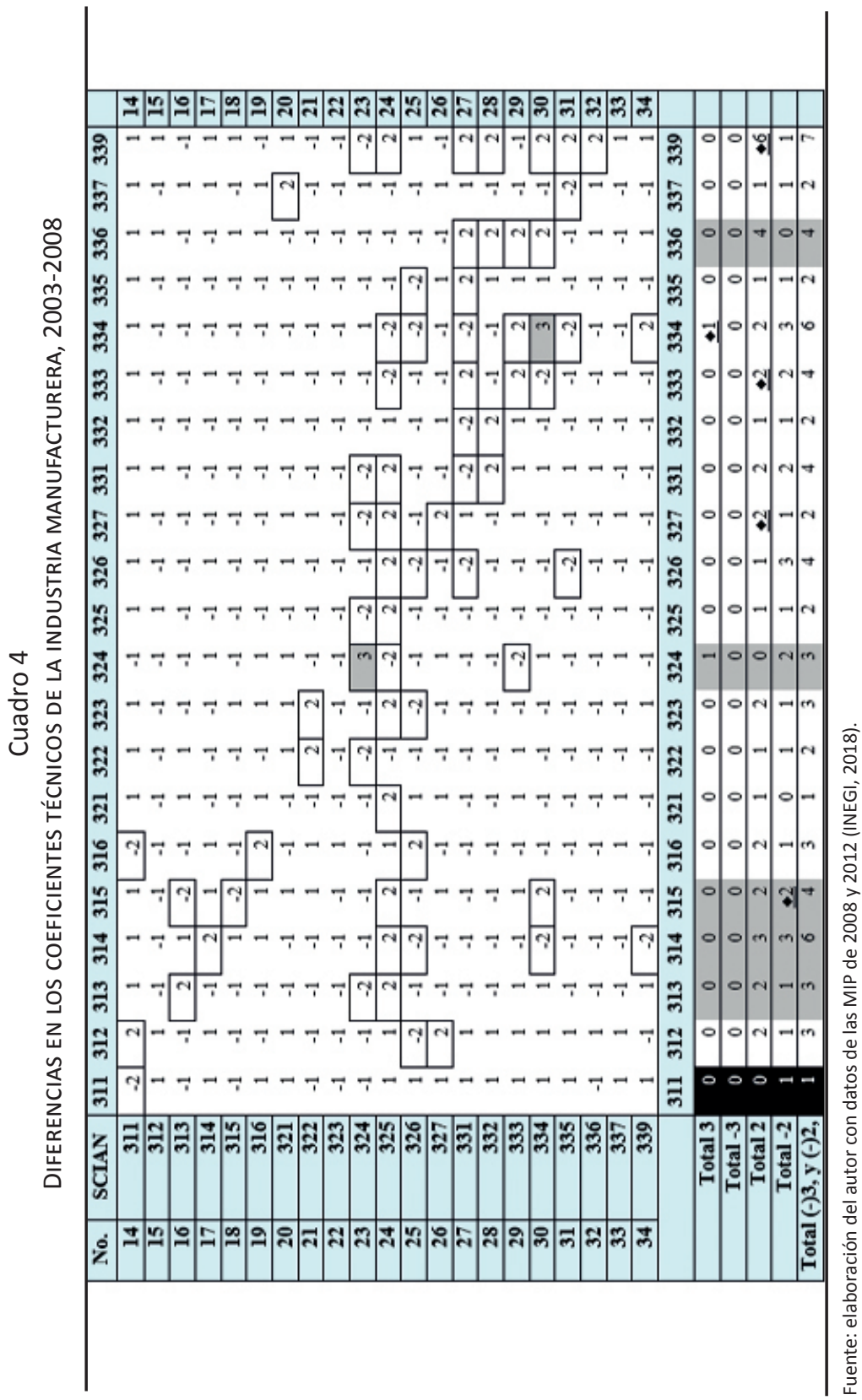


Respecto al subsector otras industrias manufactureras (339), el cuadro 4 permite observar que el crecimiento en su coeficiente de consumo intermedio se explica por el uso más intensivo de diversos insumos provenientes de la industria química (325), industrias metálicas básicas (331), fabricación de productos metálicos (332), fabricación de equipo de computación, comunicación, etc. (334), accesorios y aparatos eléctricos (335) y equipo de transporte (336). Lo anterior indica que los productos del subsector (339) aumentaron su contenido de insumos manufactureros entre 2003 y 2008, específicamente de materiales metálicos, equipo de computación, aparatos eléctricos y de transporte. Al igual que en el caso anterior, el mayor uso de este tipo de insumos justifica la reducción en el uso del factor trabajo.

Por otro lado, de los tres casos con la mayor variación en el coeficiente de consumo intermedio encontramos al subsector fabricación de prendas de vestir (315), que a diferencia de los dos casos estudiados anteriormente, mostró una reducción en el consumo de insumos intermedios. Esto se debe principalmente a la reducción de media magnitud en el consumo de insumos textiles (313) y de insumos provenientes del mismo sector (315); lo cual acompañado de una reducción en el coeficiente de empleo indica un uso más eficiente de los insumos primarios como consecuencia de un mayor uso de capital.

También se puede ver que entre 2003 y 2008 la distribución (vectores fila) de los productos de los subsectores fabricación de productos derivados del petróleo (324), industria química (325), industria del plástico y del hule (326) e industrias metálicas básicas (331) ha sufrido los cambios más relevantes (son las filas con mayor número de variaciones medias y altas). De manera específica, vemos que la distribución de los productos de los subsectores (324) y (326) se ha visto en su mayoría reducida, esto sugiere que ha disminuido la intensidad con la que el resto de subsectores manufactureros hacen uso de estos insumos. La razón de este cambio puede deberse al reciente uso de energías verdes y sustentables y al menor consumo de plástico. Además, las últimas filas del cuadro 4 indican que los subsectores con cambio técnico tipo 1 (celdas sombreadas en blanco) muestran en su mayoría un elevado número de variaciones positivas de magnitud media y alta, lo cual justifica el incremento en el coeficiente de consumo intermedio total de estos subsectores (ver gráfica 2). 
Respecto al periodo 2008-2012, se observa que el número de coeficientes por subsector que mostraron una variación de magnitud media y alta se redujo en comparación con los del primer periodo (ver anexo $3)$. De hecho, se aprecia que ningún coeficiente técnico mostró una variación de magnitud alta (3 o -3). Del análisis de la sección anterior, encontramos que en este periodo, los subsectores con el mayor cambio en su coeficiente de consumo intermedio son: industria química (325), fabricación de maquinaria y equipo (333) y fabricación de accesorios $y$ aparatos eléctricos (335). El cambio en el primero de estos subsectores se caracteriza por la reducción en el consumo de insumos intermedios, mientras que en los últimos dos por un mayor uso de este tipo de insumos.

Respecto a la industria química (325), la información del cuadro 5 indica que la reducción en el coeficiente de consumo intermedio se explica principalmente por el menor uso de los insumos provenientes del subsector de extracción de petróleo y gas (211), de insumos provenientes del mismo subsector (325) y del comercio (431). Esta situación se vio compensada con un mayor uso del factor trabajo, es decir, de 2008 a 2012 los productos de la industria química redujeron su contenido de petróleo, gas, químicos y comercio por un uso más intensivo de trabajo. Esto sugiere mayor especialización en el uso de combustibles fósiles pero una posible reducción en la productividad laboral.

Por su parte, el crecimiento en el coeficiente de consumo intermedio del subsector fabricación de maquinaria y equipo (333) se explica por el incremento de magnitud media en 5 de sus coeficientes técnicos. Este subsector aumentó sus requerimientos de los productos proveídos por los subsectores fabricación de equipo de computación y comunicación (334), por sí mismo (333), fabricación de accesorios y aparatos eléctricos (335), equipo de transporte (336) y del comercio (431). El mayor uso de este tipo de insumos sugiere una técnica de producción mucho más tecnificada en el sentido de que requiere en mayor medida insumos con un nivel tecnológico medio y alto (ver Lall, 2000); esta mayor tecnificación se acompañó y tuvo como consecuencia una reducción en el uso del factor trabajo.

En situación un tanto similar se encuentra el subsector de fabricación de accesorios y aparatos eléctricos (335) que entre 2008 y 2012 mostró un crecimiento moderado en su coeficiente de consumos intermedio, el 


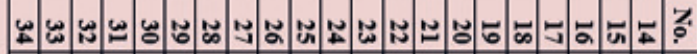

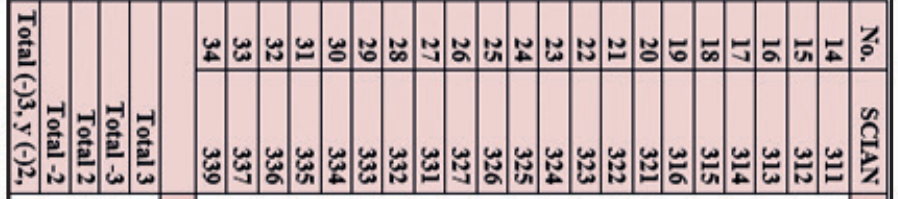
. 00000苗|-

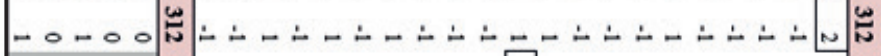

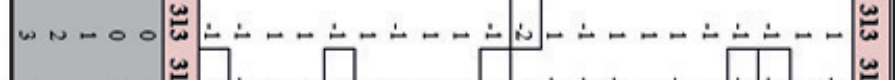
a w wo o o

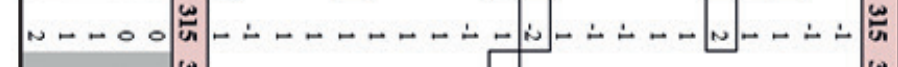

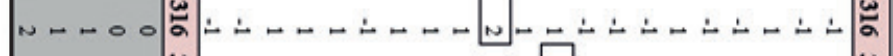
+ - 000 芯-

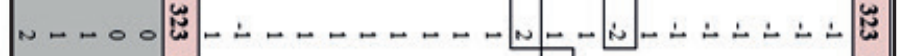
WN-O + n- - O O

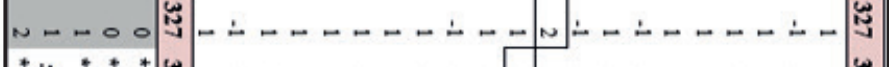
*

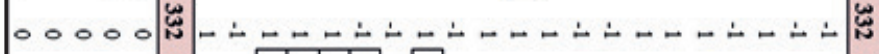
ar

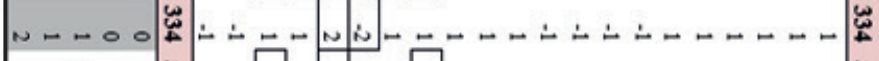

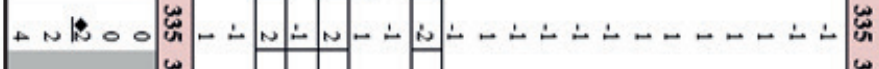
+nno o n

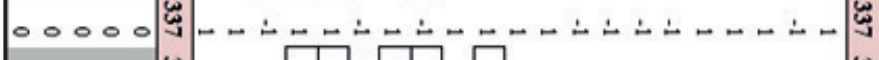

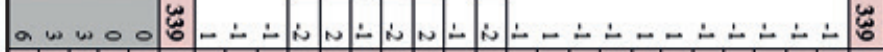


cuál se explica por un uso más intensivo de los productos provenientes de los subsectores fabricación de equipo de computación y comunicación (334) y equipo de transporte (336), este cambio también se reflejó en un menor uso del factor trabajo, esto podría sugerir que el coeficiente de capital, al igual que el de consumo intermedio, se incrementó. Por último se puede ver que en ambos periodos, la distribución del producto del subsector comercio (431) (coeficientes técnicos de la fila 431) es una de las que ha experimentado los cambios más elevados. Existen sectores que intensifican el uso del factor comercio mientras que hay otros que lo han reducido. Aunque 6 subsectores manufactureros han reducido de manera importante su uso.

\section{EFECTOS SUSTITUCIÓN Y FABRICACIÓN}

Si vamos más allá de la técnica de producción y observamos las relaciones tanto de oferta como de demanda de cada uno de los subsectores podemos tener una idea más amplia del cambio en las relaciones de la manufactura. Para ello, es necesario observar no solo las columnas de las matrices de diferencias, también las filas.

De acuerdo con Leontief (1951) y Stone (1961), los coeficientes de una matriz tienden a cambiar en el tiempo en forma bi-proporcional, por lo que los cambios en los coeficientes técnicos se deben a dos efectos, el sustitución y el fabricación. El primero opera sobre las filas y mide la importancia de ahorrar en ciertos materiales más que en otros, registra la medida en que el producto del i-ésimo sector ha sido reemplazado por, o usado como sustituto de otros productos sectoriales en la producción. Por su parte, el efecto fabricación opera sobre las columnas y mide el ahorro en insumos intermedios en relación con el uso de los factores de producción primarios, registra la medida en que la proporción de insumos intermedios tiende a reducirse en el sector j-ésimo. En ese sentido, se vuelve relevante analizar los 21 vectores fila y columna de la industria manufacturera.

De las matrices de diferencias con elementos $d_{\mathrm{ij}}$ es posible registrar la media de los coeficientes fuera de la diagonal, positivos y negativos de aquellos cuya diferencia en valor absoluto es mayor a $0.0001^{6}$ (véase Östblom, 1989) de la siguiente manera:

6 Con la finalidad de simplificar el análisis, se considera que solo los coeficientes en los que las diferencias en valor absoluto sean mayores a 0.0001 representan un cambio representativo del coeficiente. 


$$
\begin{gathered}
e=\frac{\sum_{i}^{n} \sum_{j}^{n} d_{i j}}{(n(n-1)-k)} \quad i \neq j ; \quad k=\text { número de }\left|d_{i j}\right| \leq 0.0001 \\
e_{j}=\frac{\sum_{i}^{n} d_{i j}}{\left((n-1)-k_{j}\right)} \quad i \neq j ; \quad k_{j}=\text { número de }\left|d_{i j}\right| \leq 0.0001 \text { en la columna } j \\
e_{i}=\frac{\sum_{j}^{n} d_{i j}}{\left((n-1)-k_{i}\right)} \quad i \neq j ; \quad k_{j}=\text { número de }\left|d_{i j}\right| \leq 0.0001 \text { en la fila } i
\end{gathered}
$$

Donde: $i, j$ representan a los 21 subsectores manufactureros que van desde el subsector 311 (posición 14) hasta el 339 (posición 34), e es la media de todas las diferencias de los coeficientes fuera de la diagonal que son mayores en valor absoluto a 0.0001 ; la media $e_{j}$ es la media de las diferencias de los coeficientes fuera de la diagonal que se encuentran en la columna $j$ de las matrices $\mathrm{D}_{1}$ y $\mathrm{D}_{2}$ y la media $e_{i}$ registra el valor medio de las diferencias de los elementos fuera de la diagonal que se encuentran en la fila $i$ de las matrices $\mathrm{D}_{1} \mathrm{y}_{2}$. La dirección del cambio en la técnica entre dos periodos se indica por la media $e$. Si esta media es negativa, los coeficientes técnicos del último año son en promedio menores que los del año precedente, lo que podría sugerir un menor uso generalizado de insumos intermedios, es decir, un cambio técnico que mejora la eficiencia productiva. El valor de las medias $e_{i}$ y $e_{j}$ que se encuentran en la parte baja del cuadro 6 indican la contribución de los coeficientes técnicos de una fila o una columna al cambio técnico. Del análisis de las medias $e_{i}$ y $e_{j}$ podremos concluir si el cambio en la técnica se ha dado por el efecto sustitución o por el efecto fabricación (Östoblom, 1989).

El cuadro 6 muestra para los periodos 2003-2008 y 2008-2012 la distribución de la media de las diferencias en las filas y columnas de los subsectores manufactureros según el signo y la magnitud de la variación. Los números contenidos en el cuadro reflejan el número de subsectores manufactureros en cada categoría, y en la parte baja se muestran los valores de las medias totales.

Se encuentra que, en ambos casos, es mayor el número de vectores columna que mostró una variación media negativa. En el primer periodo, 12 de los 21 subsectores manufactureros mostraron en promedio una reducción en su media $e_{j}$; mientras que en el segundo periodo 11 de los 21 mostraron una reducción. Dicho de otro modo, la mayoría de las celdas contenidas en los vectores columna de la industria manufacturera mostraron una reducción en su valor. Esto quiere decir que en ambos periodos el cambio técnico se ha dado en su mayoría por 
el efecto fabricación, el cual indica una tendencia hacia el ahorro de insumos intermedios, en la sección 2.1 se observó con mayor detalle este cambio, aunque con mayor fuerza en el periodo 2008-2012.

Cuadro 6

MEDIA DE LAS DIFERENCIAS EN LOS COEFICIENTES POR FILAS Y COLUMNAS

\begin{tabular}{|c|c|c|c|c|c|c|c|c|}
\hline \multirow[t]{3}{*}{ Categoria } & \multicolumn{4}{|c|}{ Diferencia 2003-2008 } & \multicolumn{4}{|c|}{ Diferencia 2008-2012 } \\
\hline & \multicolumn{2}{|c|}{ Por columna } & \multicolumn{2}{|c|}{ Por fila } & \multicolumn{2}{|c|}{ Por columna } & \multicolumn{2}{|c|}{ Por fila } \\
\hline & $e_{j}$ & $-e_{j}$ & $e_{i}$ & $-e_{i}$ & $e_{j}$ & $-e_{j}$ & $e_{i}$ & $-e_{i}$ \\
\hline $0.00701-0.008$ & 0 & 0 & 0 & 1 & 0 & 0 & 0 & 0 \\
\hline $0.00601-0.007$ & 0 & 1 & 0 & 0 & 0 & 0 & 0 & 0 \\
\hline $0.00501-0.006$ & 0 & 0 & 0 & 0 & 0 & 0 & 0 & 0 \\
\hline $0.00401-0.005$ & 0 & 0 & 1 & 0 & 0 & 0 & 4 & 0 \\
\hline $0.00301-0.004$ & 0 & 0 & 1 & 1 & 0 & 0 & 1 & 0 \\
\hline $0.00201-0.003$ & 0 & 0 & 0 & 1 & 1 & 1 & 1 & 1 \\
\hline $0.00101-0.002$ & 1 & 3 & 2 & 2 & 0 & 2 & 1 & 1 \\
\hline $0.0001-0.001$ & 8 & 7 & 6 & 6 & 7 & 6 & 3 & 4 \\
\hline$<0.0001$ & 0 & 1 & 0 & 0 & 2 & 2 & 3 & 2 \\
\hline \multirow[t]{2}{*}{ Total $=$} & 9 & 12 & 10 & 11 & 10 & 11 & 13 & 8 \\
\hline & $e=$ & \multicolumn{3}{|c|}{-0.000000000084} & $e=$ & \multicolumn{3}{|c|}{0.000000000074} \\
\hline \multicolumn{2}{|c|}{ Media para $e_{j}$ : } & \multicolumn{2}{|c|}{-0.00053} & \multicolumn{2}{|c|}{ Media para $e_{j}$ : } & -0.000 & & \\
\hline \multicolumn{2}{|c|}{ Media para $e_{i}$ : } & \multicolumn{2}{|c|}{-0.00025} & \multicolumn{2}{|c|}{ Media para $e_{i}$ : } & 0.001 & & \\
\hline
\end{tabular}

Fuente: elaboración del autor con datos de las MIP de 2003, 2008 y 2012 (INEGI, 2018).

Para la media por fila $e_{i}$, encontramos que en el primer periodo los vectores con medias negativas también fueron mayores, sin embargo, para el segundo periodo ésta tendencia se revirtió. Esto indica un efecto sustitución importante entre 2003 y 2008. Este cambio en la técnica de producción responde a que los insumos requeridos por otras empresas tienden a cambiar según la evolución de los productos. En el primer periodo 11 de los 21 subsectores mostraron una reducción en el valor medio de sus coeficientes por fila, en el segundo periodo 8 de 21 se encontraron en esta situación.

Por último, la información contenida en el cuadro 6 deja ver que, para el periodo 2003-2008, el valor medio de todos los coeficientes e tiende a ser ligeramente negativo, lo que indica que los coeficientes manufactureros de 2008 tienden a ser menores a los de 2003. Esto se 
refuerza por el hecho de que para el mismo periodo, los valores medios por fila y por columna son menores en 2008 que en 2003.

Para el segundo periodo, la situación es diferente ya que el valor medio e de todos los coeficientes es ligeramente positivo, lo cuál se explica porque el cambio positivo en los coeficientes por fila ha superado el cambio negativo observado en las columnas, por ello se concluye que en promedio los coeficientes de la matriz de 2012 son ligeramente mayores a los de la matriz de 2008. El hecho de que el cambio técnico se defina por el efecto fabricación deja ver la importancia en la relación entre el uso de los insumos intermedios y los primarios.

\section{CONCLUSIONES}

En este documento se realizan diversos ejercicios con la finalidad de medir el cambio en la técnica de producción de los subsectores manufactureros. El análisis del cambio técnico mediante la metodología de insumo-producto requiere el uso de las matrices totales, y que estas estén valuadas a un mismo nivel de precios. La técnica de producción de los sectores manufactureros se representa en la matriz de insumoproducto ya que muestra el conjunto de los factores de producción que se combinan durante el proceso así como las cantidades a utilizar de cada uno. La medición del cambio en la técnica se puede realizar en dos etapas. En la primera, se identifica el cambio en la intensidad con la que se utilizan los insumos intermedios en comparación con los insumos primarios, y en la segunda se analiza el cambio en el uso de los diferentes insumos intermedios.

En conjunto los resultados muestran que entre 2003 y 2008 y después entre 2008 y 2012 hubo un cambio importante en la intensidad con la que se utiliza los insumos primarios (principalmente el empleo) en relación con los insumos intermedios. Sin embargo, también se encontró que los coeficientes técnicos de los insumos intermedios tienden a ser muy estables en los periodos de mediano plazo. Esto indica que a mediano plazo, el cambio técnico se observa en la intensidad de uso del empleo, mientras que muy probablemente, en el largo plazo el cambio técnico se explique por el cambio en los coeficientes técnicos intermedios.

Respecto a la primera etapa, para el periodo completo resulta evidente que la gran mayoría de los subsectores aplicó un cambio técnico ahorrador de empleo, esto se observa porque los tipos de cambio 
técnico más frecuentes fueron el 2 y 3 , cuya característica en común es un menor coeficiente de empleo. Esto sin lugar a dudas debería reflejarse en un incremento en la productividad laboral, relación cuyo estudio queda pendiente para trabajos posteriores.

La medición del cambio técnico por etapas permitió obtener información específica para cada uno de los subsectores. Entre 2003 y 2008 destacó el cambio técnico de los subsectores fabricación de equipo de computación, comunicación, etc. (334) y otras industrias manufactureras (339) que claramente muestran una tendencia a la tecnificación y mejora de los procesos productivos, y a la sustitución del empleo por insumos con mayor contenido tecnológico.

Se encontró que en el periodo 2003-2008 la mayoría de los coeficientes de consumo intermedio mostró una variación positiva, pero que el valor promedio de las diferencias de los coeficientes técnicos relacionados a la industria manufacturera mostró una tendencia a la baja. Esto se explica porque la magnitud en la que aumentaron algunas de las celdas fue superior a la reducción del resto de coeficientes del subsector en cuestión, es decir, en la mayoría de los casos, la variación en el coeficiente de consumo intermedio se determinó por la variación de magnitud media y alta de un número reducido de celdas.

Adicionalmente, el hecho de que los coeficientes técnicos de la industria manufacturera de 2008 sean ligeramente menores a los de 2003 tanto por fila como por columna implica una ligera reducción en la intensidad de uso de los insumos intermedios.

En el periodo 2008-2012 se encontró que los subsectores fabricación de maquinaria y equipo (333) y fabricación de accesorios y aparatos eléctricos (335) mostraron un aumento en sus requerimientos de insumos más tecnificados, esto tuvo como consecuencia una reducción en el uso del empleo. En este periodo también sobresale el caso de la industria química (325) que redujo de manera importante el uso de combustibles fósiles pero incremento el uso del empleo.

Por su parte, entre 2008 y 2012, la mayoría de los subsectores experimentó una reducción en el uso de los insumos intermedios, o dicho de otro modo, un aumento en el uso de insumos primarios. En cuanto a los cambios en los coeficientes técnicos, se observa que estos fueron de menor magnitud. Además este periodo se caracteriza por tener un valor medio de las diferencias en los coeficientes de la manufactura ligera- 
mente positivo, y que se explica porque el valor medio por columna fue negativo pero positivo por fila.

Por último, se encontró que en ambos periodos el cambio técnico se ha dado en su mayoría por el efecto fabricación, hecho que deja ver la importancia de la relación entre el uso de insumos intermedios e insumos primarios. En conclusión, la información sugiere que entre 2003 y 2008 el cambio técnico mostró una tendencia hacia el ahorro de insumos primarios, lo que significa que la magnitud en la que aumentaron algunos coeficientes contrarrestó la disminución generalizada en los coeficientes de la manufactura. En cambio, entre 2008 y 2012 se observa un cambio técnico con tendencia al ahorro de insumos intermedios; aunque en promedio los coeficientes de 2012 son ligeramente mayores a los de 2008, la magnitud de la reducción en los coeficientes parece ser mayor que la magnitud del aumento.

\section{Biblografía}

Arrow, K. (1962). "The Economic Implications of Learning by Doing". Review of Economic Studies. 29 (3): 155-73.

Carter, A. (1970). Structural Change in the American Economy. Harvard University Press. Cambridge Massachusetts.

Coombs, R., Saviotti, P. y V. Walsh. (1987) Economics and Technological Change. Rowman and Littlefield. United States.

De Long, J. y L. Summers (1991). "Equipment Investment and Economic Growth." The Quarterly Journal of Economics. May. 445-502.

Dosi, G. (1982). "Technological paradigms and technological trajectories. A suggested interpretation of the determinants and directions of technological change". Science Policy Research Unit, University of Sussex, Brighton, U.K.

Dowrick, S. (1994) "Investment and Resource Allocation as Sources of LongRun Growth". En Fagerberg, J., Verspagen B. y N. von Tunzelmann (coord.) The Dynamics of Technology, Trade and Growth. Pp. 99-122. Great Britain.

Dussel-Peters, E. (2003). "Características de las actividades generadoras de empleo en la economía mexicana (1988-2000)". Investigación Económica, 62(243), 123-154.

Dussel Peters, E. y Ortiz, S. (2013). “Tendencias macroeconómicas”. Monitor de la Manufactura Mexicana, 9(10), 13-29. 
Freeman, C. y C. Perez (1988), "Structural crises of adjustment: business cycles and investment behaviour", En: G. Dosi, et al. (eds.) Technical Change and Economic Theory, London: Francis Pinter, pp. 38-66.

Fujii-Gambero, G. y R. Cervantes (2013). "México: Valor agregado en las exportaciones manufactureras" Revista CEPAL, Núm. 109, 143-158

INEGI (2008). "Sistema de Clasificación Industrial de América del Norte (SCIAN), 2007". Instituto Nacional del Estadística y Geografía

INEGI (2018). “Matrices de insumo-producto, 2003, 2008 y 2012”. Instituto Nacional del Estadistica y Geografia. https:/www.inegi.org.mx/temas/ $\mathrm{mip} /$

Kaldor, N. (1966). "Causes of the Slow Rate of Economic Growth of the United Kingdom”. Cambridge University Press.

Kim, L. (1997). Imitation to innovation: the dynamics of Korea's technological learning, Harvard Business School Press, Boston

Kondratieff, N. (1935). "The Long Waves in Economic Life", The Review of Economics and Statistics, Vol. 1, No. 6, 105-115.

Lall, S. (1992). "Technological capabilities and industrialization", World Development, Vol. 20, 165-186.

Lall, S. (2000). "The technological structure and performance of developing country manufactures exports, 1985-1998". Oxford Development Studies, 28, 337-369.

Lall, S. (2004). "Technology and industrial development in an era of globalization" en Chang (ed.). Rethinking development economics, London

Leontief, W. (1951). The structure of the American economy, 1919-1939. Oxford University Press. New York, USA

Molins Codina, J. (1973). "Progreso tecnológico, progreso técnico y desarro1lo. Una aplicación al caso español." Cuadernos de economía Número 1. Universidad de Barcelona

Murillo-Villanueva, B., Puchet Anyul, M. y G. Fujii-Gambero. (en prensa). "Actualización de la matriz total de insumo-producto de México de 2003. Aplicación de los métodos de doble deflación y RAS”. Realidad, Datos y Espacio. Revista Internacional de Estadística y Geografía.

Östblom, G. (1989). "Change in technical structure of the Swedish Economy." Working Paper 2, Konjunkturinsitutet/Ekonomiska ráldet, Stockholm.

Pasinetti, L. (1983) "The Accumulation of Capital". Cambridge Journal of Economics. Vol. 7, pp. 405-411. Reino Unido.

Prebisch R. (1949). El desarrollo económico de América Latina y algunos de sus principales problemas, E/CN.12/89, Santiago de Chile

Rosenberg, N. (2000) Schumpeter and the Endogeneity of Technology. Some American Perspectives. The Graz Schumpeter Lectures. Routledge. New York. 
Ruiz-Nápoles, P. (2004): "Exports, growth and employment in Mexico, 19782000”, Journal of Post Keynesian Economics, 27(1), 105-124

Sánchez, I. (2012). "Ralentización del crecimiento y manufacturas en México". Nóesis, Revista de Ciencias Sociales y Humanidades, 21(41), 137-170.

Schumpeter, J. (1942) Capitalism, Socialism and Democracy. Routledge. United Kingdom.

Solow, R. (1956) "A Contribution to the Theory of Economic Growth", Quarterly Journal of Economics, 70, 1. Febrero: 65-94.

Stone, R. (1961). Input-Output and National Accounts. Paris: Organization for Economic Cooperation and Development.

Vaccara, B. (1970). "Changes over time in input-output coefficients for the United States." In: Carter, A. and A. Brody (eds.) Contributions to InputOutput Analysis. North-Holland Publishing Co., Vol. 2

\section{Anexo 1. CLASIFICACIÓn SCIAN, 2017}

\begin{tabular}{|c|c|c|c|c|c|}
\hline No. & SCIAN & Descripción & No. & SCIAN & Descripción \\
\hline 1 & 111 & Agricultura & 40 & 485 & $\begin{array}{c}\text { Transporte terrestre de pasajeros, } \\
\text { excepto por ferrocarril }\end{array}$ \\
\hline 2 & 112 & Cría y explotación de animales & 41 & 486 & Transporte por ductos \\
\hline 3 & 113 & Aprovechamiento forestal & 42 & 487 & Transporte turístico \\
\hline 4 & 114 & Pesca, caza y captura & 43 & 488 & $\begin{array}{l}\text { Servicios relacionados con el } \\
\text { transporte }\end{array}$ \\
\hline 5 & 115 & $\begin{array}{c}\text { Servicios relacionados con las } \\
\text { actividades agropecuarias y forestales }\end{array}$ & 44 & 491 & Servicios postales \\
\hline 6 & 211 & Extracción de petróleo y gas & 45 & 492 & Servicios de mensajería y paquetería \\
\hline 7 & 212 & $\begin{array}{l}\text { Minería de minerales metálicos y no } \\
\text { metálicos, excepto petróleo y gas }\end{array}$ & 46 & 493 & Servicios de almacenamiento \\
\hline 8 & 213 & Servicios relacionados con la minería & 47 & 511 & $\begin{array}{c}\text { Edición de periódicos, revistas, libros, } \\
\text { software y otros materiales, }\end{array}$ \\
\hline 9 & 221 & $\begin{array}{c}\text { Generación, transmisión y distribución } \\
\text { de energía eléctrica }\end{array}$ & 48 & 512 & $\begin{array}{l}\text { Industria fímica y del video, e industria } \\
\text { del sonido }\end{array}$ \\
\hline 10 & 222 & $\begin{array}{l}\text { Suministro de agua y suministro de gas } \\
\text { por ductos al consumidor final }\end{array}$ & 49 & 515 & Radio y televisión \\
\hline 11 & 236 & Edificación & 50 & 517 & Otras telecomunicaciones \\
\hline 12 & 237 & Construcción de obras de ingeniería civil & 51 & 518 & $\begin{array}{l}\text { Procesamiento electrónico de } \\
\text { información, hospedaje y otros } \\
\text { servicios relacionados }\end{array}$ \\
\hline 13 & 238 & $\begin{array}{l}\text { Trabajos especializados para la } \\
\text { construcción }\end{array}$ & 52 & 519 & Otros servicios de información \\
\hline 14 & 311 & Industria alimentaria & 53 & 521 & Banca central \\
\hline
\end{tabular}




\section{ANEXo 1. CLASIFICACIÓN SCIAN, 2017 (CONTINUACIÓN)}

\begin{tabular}{|c|c|c|c|c|c|}
\hline 15 & 312 & Industria de las bebidas y del tabaco & 54 & 522 & $\begin{array}{l}\text { Instituciones de intermediación } \\
\text { crediticia y financiera no bursátil }\end{array}$ \\
\hline 16 & 313 & $\begin{array}{l}\text { Fabricación de insumos textiles y } \\
\text { acabado de textiles }\end{array}$ & 55 & 523 & $\begin{array}{l}\text { Actividades bursátiles, cambiarias y de } \\
\text { inversión financiera }\end{array}$ \\
\hline 17 & 314 & $\begin{array}{l}\text { Fabricación de productos textiles, } \\
\text { excepto prendas de vestir }\end{array}$ & 56 & 524 & $\begin{array}{c}\text { Compañías de fianzas, seguros y } \\
\text { pensiones }\end{array}$ \\
\hline 18 & 315 & Fabricación de prendas de vestir & 57 & 531 & Servicios inmobiliarios \\
\hline 19 & 316 & $\begin{array}{l}\text { Curtido y acabado de cuero y piel, y } \\
\text { fabricación de productos de cuero, piel y } \\
\text { materiales sucedáneos }\end{array}$ & 58 & 532 & Servicios de alquiler de bienes muebles \\
\hline 20 & 321 & Industria de la madera & 59 & 533 & $\begin{array}{l}\text { Servicios de alquiler de marcas } \\
\text { registradas, patentes y franquicias }\end{array}$ \\
\hline 21 & 322 & Industria del papel & 60 & 541 & $\begin{array}{c}\text { Servicios profesionales, científicos y } \\
\text { técnicos }\end{array}$ \\
\hline 22 & 323 & Impresión e industrias conexas & 61 & 551 & Corporativos \\
\hline 23 & 324 & $\begin{array}{l}\text { Fabricación de productos derivados del } \\
\text { petróleo y del carbón }\end{array}$ & 62 & 561 & Servicios de apoyo a los negocios \\
\hline 24 & 325 & Industria química & 63 & 562 & $\begin{array}{l}\text { Manejo de desechos y servicios de } \\
\text { remediación }\end{array}$ \\
\hline 25 & 326 & Industria del plástico y del hule & 64 & 611 & Servicios educativos \\
\hline 26 & 327 & $\begin{array}{l}\text { Fabricación de productos a base de } \\
\text { minerales no metálicos }\end{array}$ & 65 & 621 & $\begin{array}{c}\text { Servicios médicos de consulta externa } \\
\text { y servicios relacionados }\end{array}$ \\
\hline 27 & 331 & Industrias metálicas básicas & 66 & 622 & Hospitales \\
\hline 28 & 332 & Fabricación de productos metálicos & 67 & 623 & $\begin{array}{l}\text { Residencias de asistencia social y para } \\
\text { el cuidado de la salud }\end{array}$ \\
\hline 29 & 333 & Fabricación de maquinaria y equipo & 68 & 624 & Otros servicios de asistencia social \\
\hline 30 & 334 & $\begin{array}{c}\text { Fabricación de equipo de computación, } \\
\text { comunicación, medición y de otros } \\
\text { equipos. }\end{array}$ & 69 & 711 & $\begin{array}{l}\text { Servicios artísticos, culturales } \\
\text { y deportivos, y otros servicios } \\
\text { relacionados }\end{array}$ \\
\hline
\end{tabular}

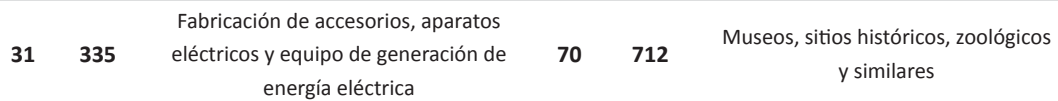

\begin{tabular}{|c|c|c|c|c|c|}
\hline 32 & 336 & Fabricación de equipo de transporte & 71 & 713 & $\begin{array}{l}\text { Servicios de entretenimiento en } \\
\text { instalaciones recreativas y otros } \\
\text { servicios recreativos }\end{array}$ \\
\hline 33 & 337 & $\begin{array}{l}\text { Fabricación de muebles, colchones y } \\
\text { persianas }\end{array}$ & 72 & 721 & Servicios de alojamiento temporal \\
\hline 34 & 339 & Otras industrias manufactureras & 73 & 722 & $\begin{array}{l}\text { Servicios de preparación de alimentos } \\
\text { y bebidas }\end{array}$ \\
\hline 35 & 431 & Comercio & 74 & 811 & $\begin{array}{l}\text { Servicios de reparación y } \\
\text { mantenimiento }\end{array}$ \\
\hline 36 & 481 & Transporte aéreo & 75 & 812 & Servicios personales \\
\hline 37 & 482 & Transporte por ferrocarril & 76 & 813 & Asociaciones y organizaciones \\
\hline 38 & 483 & Transporte por agua & 77 & 814 & Hogares con empleados domésticos \\
\hline 39 & 484 & Autotransporte de carga & 78 & 931 & $\begin{array}{c}\text { Actividades del gobierno y organismos } \\
\text { internacionales }\end{array}$ \\
\hline
\end{tabular}

Fuente: Elaboración del autor con base en INEGI (2008). 
号

$\frac{4}{4}$

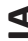

ำ

n

은

4

w

ด

$\varangle$

$\frac{2}{2}$

แั

بิ

은

는

$>$

ด

ב

z

4

4

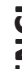

늠

N

울

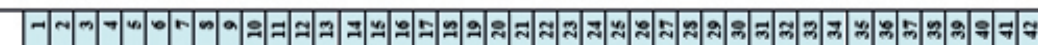

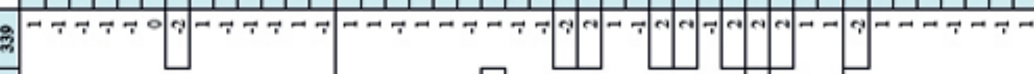

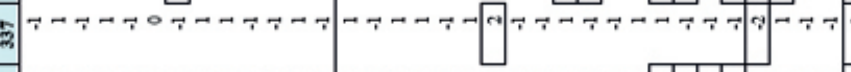

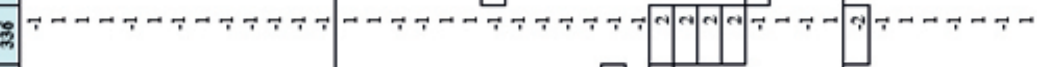

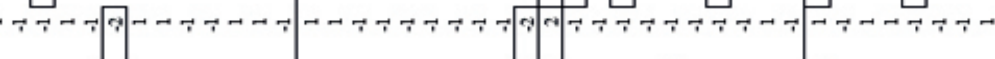

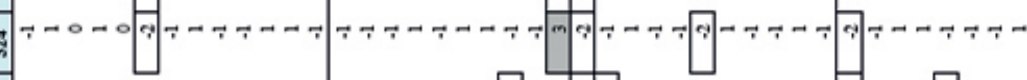




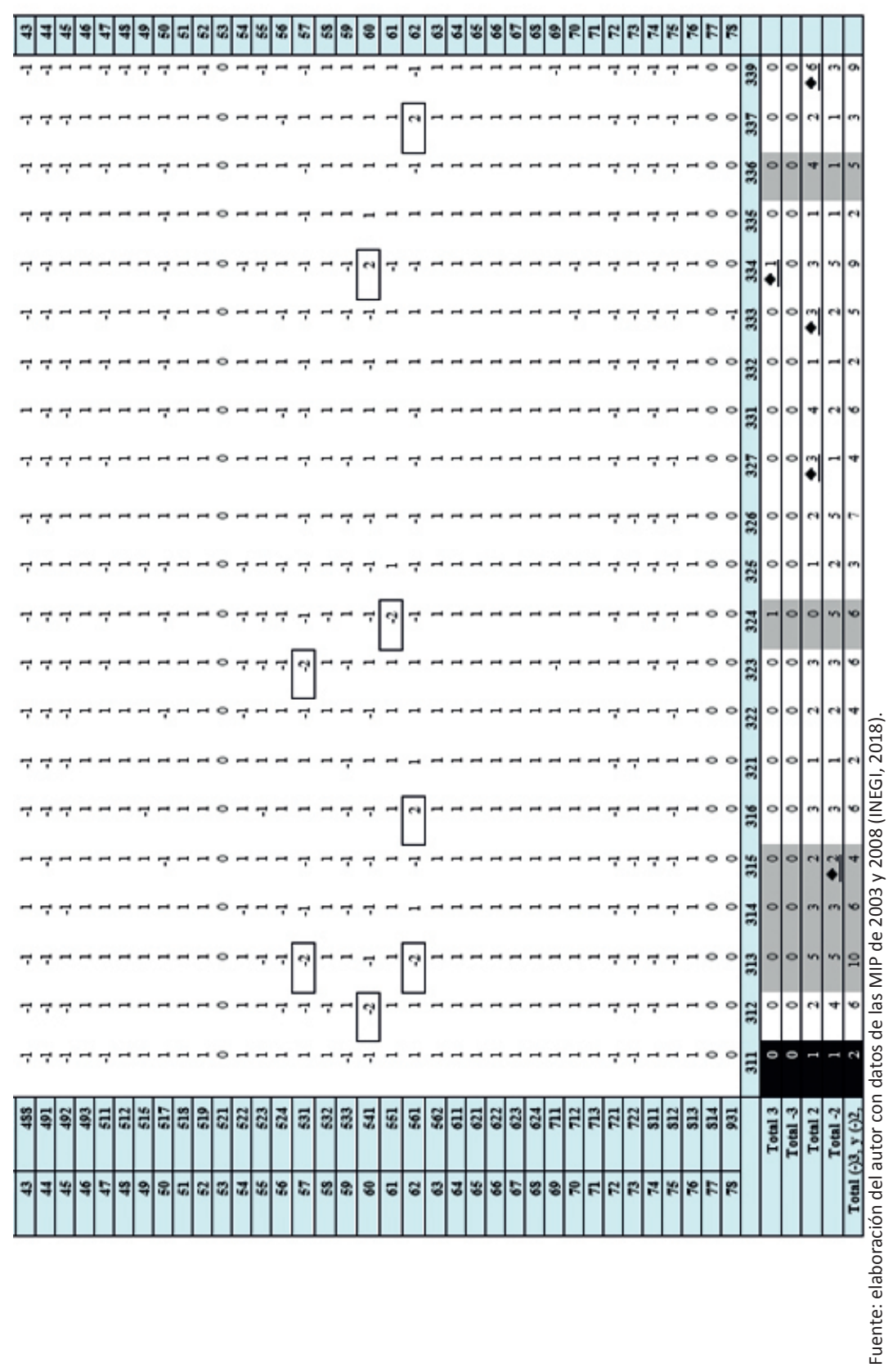




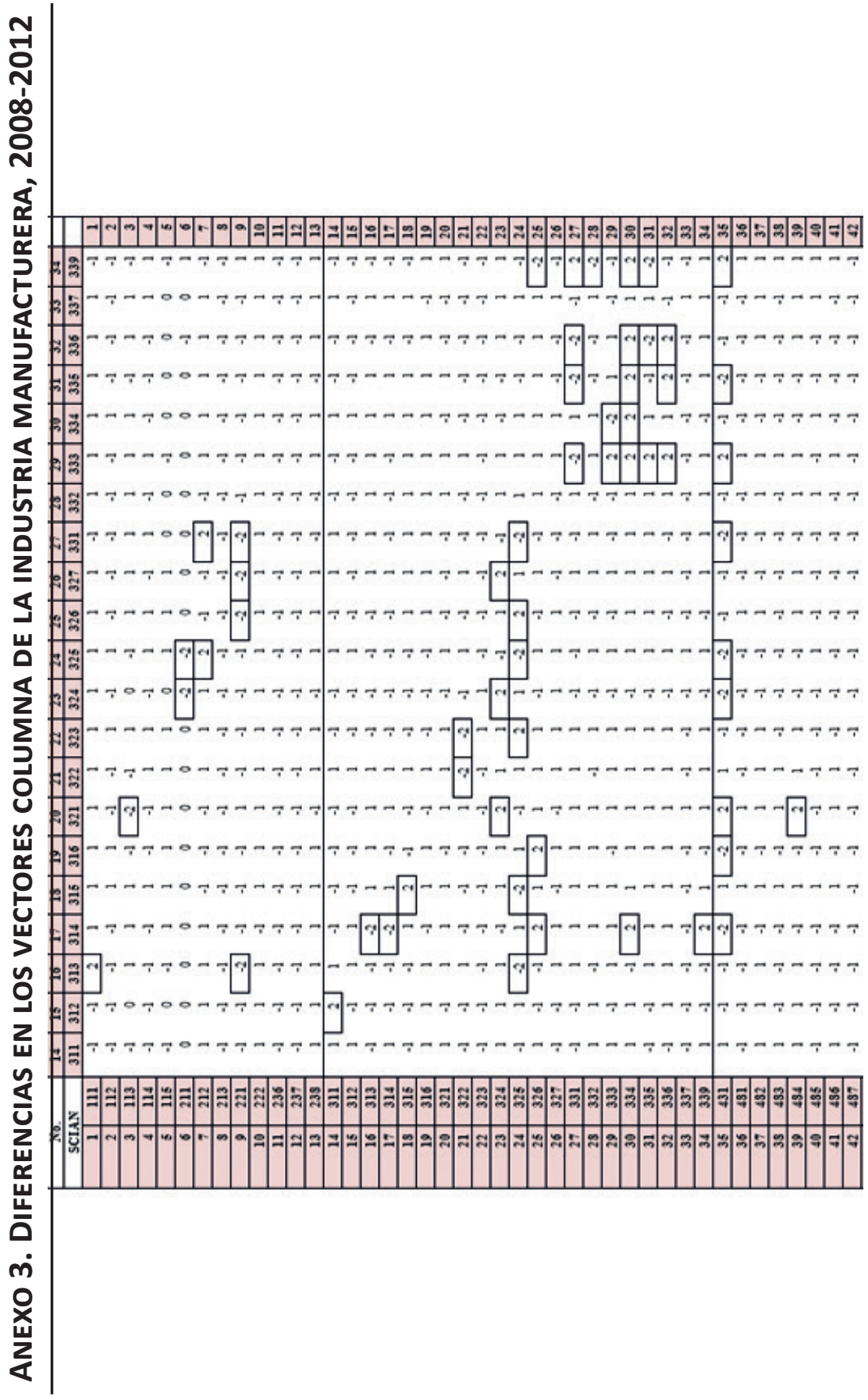




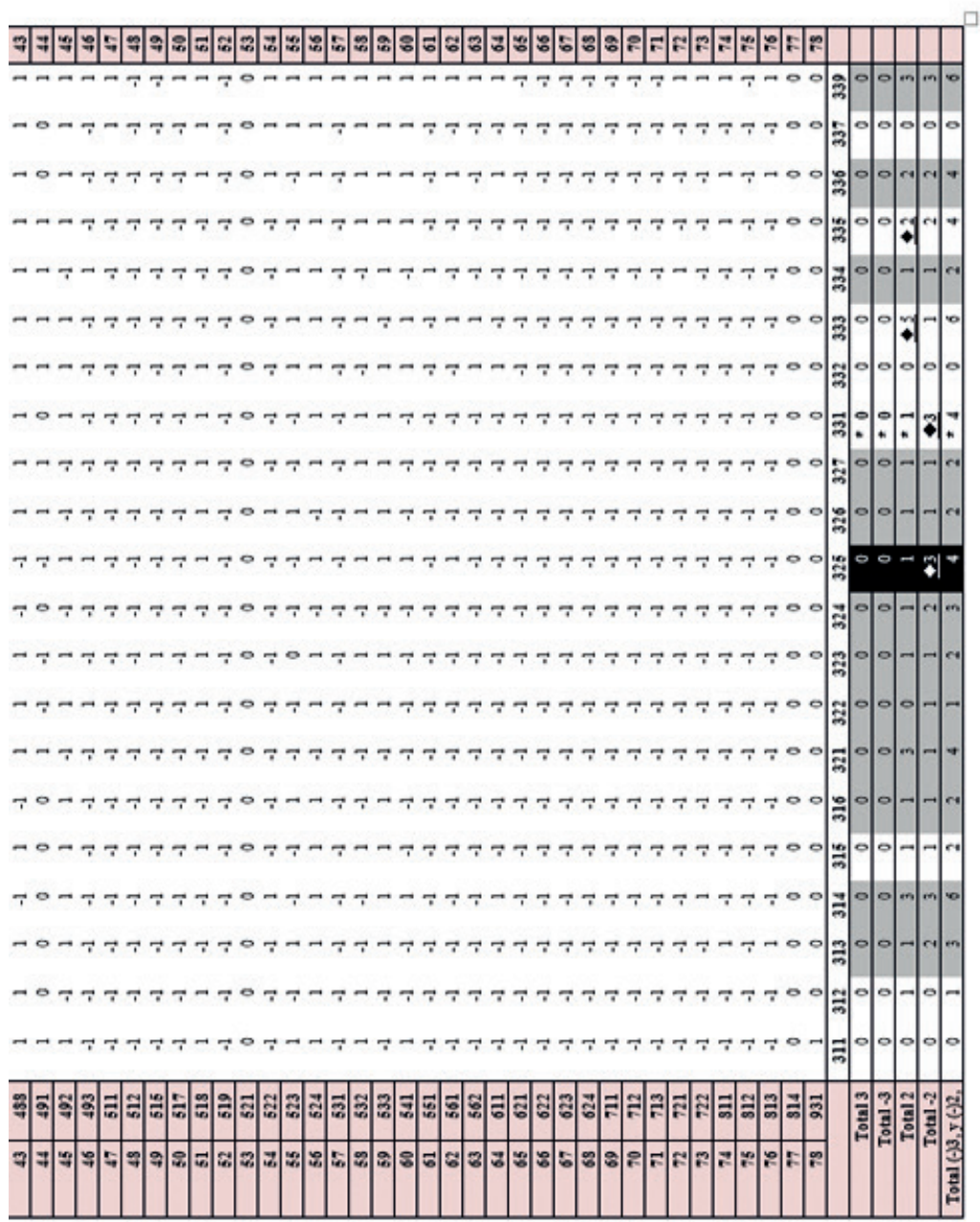

